九州大学学術情報リポジトリ

Kyushu University Institutional Repository

\title{
AN ABSTRACT RELATIONAL MODEL AND NATURAL JOIN FUNCTORS
}

Kato, Akihiko

International Institute for Advanced Study of Social Information Science, Fujitsu Limited

https://doi.org/10.5109/13349

出版情報: Bulletin of informatics and cybernetics. 20 (3/4), pp.95-106，1983-03. Research Association of Statistical Sciences

バージョン :

権利関係 : 


\title{
AN ABSTRACT RELATIONAL MODEL AND NATURAL JOIN FUNCTORS
}

\author{
By
}

\begin{abstract}
Akihiko KATO*
Abstract

A meta-model for database models called an abstract relational model which is obtained by a categorical abstraction of a relational model is proposed. This meta-model represents various database models, e.g. relational, network, hierarchical models as special cases. It is proved that a natural join is the right adjoint of a decomposition in the relational model. On the other hand, in our abstract relational model a natural join is defined as the right adjoint of a decomposition. A sufficient condition is shown for a database model to have natural joins.
\end{abstract}

\section{Introduction}

Several database models such as relational, network and hierarchical models have been investigated $[2,3,4]$. And also there are some investigations for unifications and conversions among these database models, e.g. [1]. However, these studies still adhere to actual database models and have not unified perspectives of various database models.

Category theory can be expected to give a comprehensive perspective for various theories. In fact, it has been applied to automata theory, system theory and computation theory etc. [8].

The purpose of this paper is to propose a meta-model, an abstract relational model, which is obtined by generalizing the relational database model in terms of category theory ${ }^{1)}$. This abstract represents various database models, e. g. relational, network and hierarchical models as special cases. Furthermore, it gives a framework which allows to discuss database models without going into details of concrete database models. Particularly, a natural join is defined as the right adjoint of a decomposition in our abstract relational model while a natural join is defined by going into details of tuples of relations in Codd's relational model. Furthermore, our definition of natural joins shows clearly a relationship between natural joins and decompositions, which are not always the inverses of natural joins.

As an application of the abstract relational models, a sufficient condition is shown for a database model to have natural joins.

* International Institute for Advanced Study of Social Information Science, Fujitsu Limited, Numazu, 410-03 Japan.

1) There are other studies which attempt to apply category theory to relational databases $[7,9,11]$. 
For the above purpose, we take an approach that we reformalize the relational model by the category of sets and then we define an abstract relational model by generalizing such a categorical formalization.

In Section 2, we define categories of relations by introducing morphisms between relations using the category of sets to reformalize a relational database model. Projections, decompositions and naturl joins are defined to be functors and it is proved that a natural join is the right adjoint of a decomposition. In Section 3, an abstract relational model is defined by generalizing the relational model of Section 2. A natural join is defined as the right adjoint of a decomposition functor, As an important application, it is proved that an abstract relational model has natural joins if each functor included as a constituent of this model has the right adjoint and each category of occurrences has pullbacks. Finally, we exemplify formulations of relational, network and hierarchical madels as abstract relational models.

\section{Relation Data Model on the Category of Sets}

In this section, we formalize the category of relation on each attribute set and show that a natural join is regarded as the right adjoint of a decomposition by projections.

\subsection{Category of relations and projection functors}

We define the category of relations by introducing morphisms between relations.

Definition 2.1. (Relation $R$ ) Let $A$ be a set. We call $A$ a set of attributes or an attribute set. A relation on $A$ is a triple $R=(A, D, B)$, where $D=\left\{D_{i}\right\}_{i \in A}$ is a family of sets called domains, and $B$ is a subset (called a body in this paper) of the Cartesian Product $\prod_{i \in A} D_{i}$ which is the set $\left\{t: A \rightarrow \bigcup_{i \in A} D_{i} \mid(\forall i \in A) t(i) \in D_{i}\right\}$. An element $t$ of $\prod_{i \in A} D_{i}$ is called a tuple and is described by $(t(i))_{i \in A}$.

We introduce operators $D^{A}$ and - to take out the family of domains $D$ and the body $B$ of a given relation $R$ :

$$
R=\left(A, D^{A} R, \bar{R}\right) .
$$

Furthermore, for an attribute $i(i \in A)$, the domain $D_{i}$ is denoted by $D_{i}^{A} R$ with the operator $D_{i}^{A}$.

The operator $D^{A}$ will be extended to a functor later in this section.

Definition 2.2. (morphism $f$ ) Let $Q$ and $R$ are relations on the same attribute set $A$. A morphism $f$ from $Q$ to $R$ is a family $\left\{f_{i}: D_{i}^{A} Q \rightarrow D_{i}^{A} R\right\}_{i \in A}$ of functions indexed by $A$ such that

$$
\left(\prod_{i \in A} f_{i}\right)(\bar{Q}) \subset(\bar{R}) .
$$

$\prod_{i \in A} f_{i}: \prod_{i \in A} D_{i}^{A} Q \rightarrow \prod_{i \in A} D_{i}^{A} R$ is the Cartesian product which assigns $t \in \prod_{i \in A} D_{i}^{A} Q$ to $\left(f_{i}(t(i))\right)_{i \in A}$.

We write $f: Q \rightarrow R$ or $Q \stackrel{f}{\rightarrow} R$ to indicate that the morphism $f$ is from $Q$ to $R$.

Definition 2.3. (category $\boldsymbol{R}^{A}$ ) Let $A$ be a set of attributes. $\boldsymbol{R}^{A}$ denotes the category of all the relations on $A$ and all the morphisms among these relations. The composition $g \circ f: P \rightarrow R$ of morphisms $g: Q \rightarrow R$ and $f: P \rightarrow Q$ in $\boldsymbol{R}^{A}$ is defined as $\left\{g_{i} \circ f_{i}\right\}_{i \in A}$. 
It is easy to see that $\boldsymbol{R}^{A}$ is a well-defined category.

The operator $D^{A}$ becomes a funcor

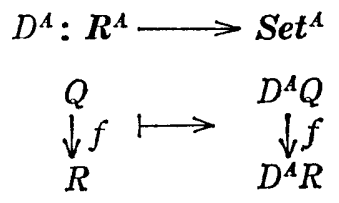

where Set is the category of sets and functions and $\boldsymbol{S e t} \boldsymbol{t}^{A}$ is the functor category from $A$ (regarded as a discrete category) to $\boldsymbol{S e t}$. $\boldsymbol{S e t}^{A}$ can be regarded as the category of $A$-indexed families of sets and $A$-indexed families of functions.

Definition 2.4. Let $A$ and $B$ be attribute sets and $a: A \rightarrow B$ be a function. Two functor $a^{*}: \boldsymbol{S e t}^{B} \rightarrow \boldsymbol{S e t}^{A}$ (the projection functor of domains on $a$ ) and $a^{(*)}: \boldsymbol{R}^{B} \rightarrow \boldsymbol{R}^{A}$ (the projection functor of relations on $a$ ) is defined $a^{2)}$

(i) for $D=\left\{D_{j}\right\}_{j \in B} \in O b \boldsymbol{S e t}^{B}, a^{*} D$ is defined as the family $\left\{D_{a(i)}\right\}_{i \in A}$.

(ii) for $g=\left\{g_{j}\right\}_{j \in B}: D \rightarrow D^{\prime} \in$ Mor $\boldsymbol{S e t}^{B}, a^{*} g$ is defined as the family $\left\{g_{a(i)}\right\}_{i \in A}$,

(iii) for $R \in O b \boldsymbol{R}^{B}, a^{(*)} R=\left(A, a^{*} D^{A} R,\left\{(t(a(i)))_{i \in A} \mid t \in \bar{R}\right\}\right)$,

(iv) for $h: Q \rightarrow R \in$ Mor $R^{B}, a^{(*)} h$ is equal to $a^{*} h$ as a family of functions.

It is easy to see the followings:

for an attribute set $A, i d_{A}^{*}=I d_{\text {Set }_{A}}$ (indentity functor) and $i d_{A}^{(*)}=I d_{R^{A}}$, for a function $a: A \rightarrow B$ between attribute sets, $a^{*} \circ D^{B}=D^{A} \circ a^{(*)}$, and for attribute sets $A, B, C$ and functions $a: A \rightarrow B, b: B \rightarrow C,(b \circ a)^{*}=a^{*} \circ b^{*}$ and $(b \circ a)^{(*)}=a^{(*)} \circ b^{(*)}$.

Fig. 2.1, shows the diagrams for understanding these statements.

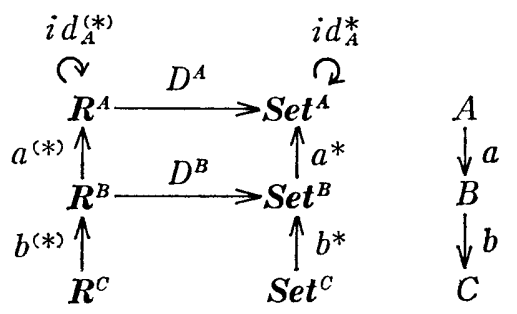

Fig. 2.1.

\subsection{Decomposition and natural join}

A natural join composes a relation of two "compatible" (or "joinable") relations. On the other hand, two projections decompose a relation into two relations which are always "compatible."

We introduce categories of compatible relations and define decomposition functors

2) In this paper, for category $\boldsymbol{C}, O b \boldsymbol{C}$ denotes the class of all the objects and Mor $\boldsymbol{C}$ denotes the class of all the morphisms of $\boldsymbol{C}$. Moreover, for objects $X$ and $Y$ of $\boldsymbol{C}, \boldsymbol{C}(X, Y)$ denotes the set of all the morphisms from $X$ to $Y$. 
and natural join functors. Finally, we show the adjointness theorem between natural joins and decompositions.

Throughout the following three definitions and a theorem. Let us suppose the diagram Fig. 2.2. for convenience, where $A, B$ and $C$ are attribute sets and $b, c$ are functions.

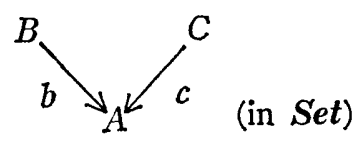

Fig. 2.2.

Definition 2.5. $\left((b, c)\right.$-compatible) For the diagram Fig. 2.2, the category $\boldsymbol{R}^{b ; c}$ of $(b, c)$-compatible relations is defined as follows.

An object is a triple $(D, P, Q)$ where $D \in O b \boldsymbol{S e t}^{A}, P \in O b \boldsymbol{R}^{B}, Q \in O b \boldsymbol{R}^{C}, D^{B} P=$ $b^{*} D$ and $D^{C} Q=c^{*} D$, i. e., the family of domains of $P$ is the projection of $D$ on $b$ and the family of domains of $Q$ is the projection of $D$ on $c$.

A morphism is a triple

$$
(g, h, k):(D, P, Q) \longrightarrow\left(D^{\prime}, P^{\prime}, Q^{\prime}\right)
$$

where $g: D \rightarrow D^{\prime}$ in $\boldsymbol{S e t}^{A}, h: P \rightarrow P^{\prime}$ in $\boldsymbol{R}^{B}, k: Q \rightarrow Q^{\prime}$ in $\boldsymbol{R}^{C}, D^{B} h=b^{*} D$ and $D^{c} k=c^{*} D$.

DEFINITION 2.6. $\left((b, c)\right.$-decomposition functor) The $(b, c)$-decomposition functor $D e c^{b ; c}$ which decomposes a relation on $A$ into $(b, c)$-compatible relations is a functor defined by

$$
\begin{aligned}
D e c^{b ; c}: & R^{A} \longrightarrow R^{b ; c} \\
& \downarrow_{R^{\prime}}^{R} f^{1 \rightarrow}\left(D^{A} R, b^{(*)} R, c^{(*)} R\right) \\
\downarrow & \left(D^{A} f, b^{(*)} f, c^{(*)} R^{\prime}, c^{(*)} R^{\prime}\right) .
\end{aligned}
$$

On the other hand, natural join functors are defined in the following.

Definition 2.7. $\left((b, c)\right.$-natural join functor) The $(b, c)$-natural join functor Join ${ }^{b ; c}$ is defined by

$$
\begin{array}{cc}
\operatorname{Join}^{b ; c}: \boldsymbol{R}^{b ; c} & \rightarrow \boldsymbol{R}^{A} \\
(D, P, Q) & P * Q \\
\downarrow(q, h, k) \mapsto & \underset{\downarrow}{\downarrow} h * k \\
\left(D^{\prime}, P^{\prime}, Q^{\prime}\right) & P^{\prime} * Q^{\prime}
\end{array}
$$

where $D^{A}(P * Q)=D, \overline{P * Q}=\left\{t \in \prod_{a \in A} D_{a} \mid\left(h_{i} t b(i)\right)_{i \in B} \in \bar{P}\right.$ and $\left(k_{j} t c(j)\right)_{j \in C} \in \bar{Q}\left(P^{\prime} * Q^{\prime}\right.$ is defined similar to $P * Q$ ) and $h * k=g$.

Fig. 2.3 shows an example of $(b, c)$-natural join. 


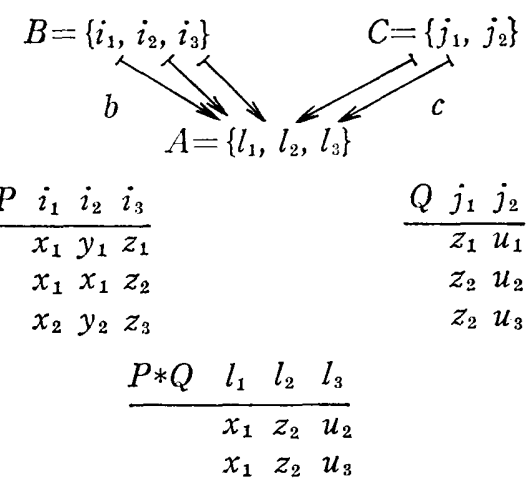

Fig. 2.3.

THEOREM 1. (Adjointness theorem between decompositions and natural joins) The natural join functor Join ${ }^{b ; c}$ is the right adjoint of the decomposition functor Dec ${ }^{b ; c}$.

$$
\boldsymbol{R}^{b ; c} \underset{\operatorname{Join}^{b ; c}}{\stackrel{D e c^{b ; c}}{\longrightarrow}} R^{A}
$$

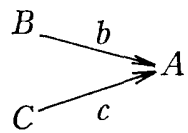

Proof. Let $R \in O b R^{A},(D, P, Q) \in O b \boldsymbol{R}^{b ; c}$. We will show there is a bijective correspondence between $\boldsymbol{R}^{b ; c}\left(\left(D^{A} R, b^{(*)} R, c^{(*)} R\right),(D, P, Q)\right)$ and $\boldsymbol{R}^{A}(R, P * Q)$.

Let $(g, h, k):\left(D^{A} R, b^{(*)} R, c^{(*)} R\right) \rightarrow(D, P, Q)$ be in $\boldsymbol{R}^{b ; c}$ and $t \in \bar{R}$. We have $\left(h_{i} t b(i)\right)_{i \in B}=\left(\prod_{i \in B} h_{i}\right)(t b(i))_{i \in B} \in \bar{P}$ since $\overline{b^{(*)} R} \equiv(t b(i))_{i \in B}$. Similarly, $\left(k_{j} t c(j)\right)_{j \in C} \in \bar{Q}$. Therefore, $t \in \overline{P * Q}$, i. e., $g$ is a morphism $R \rightarrow P * Q$ in $R^{A}$.

We can conversely show that $\left(g, b^{*} g, c * g\right)$ is a morphism $\left(D^{A} R, b^{(*)} R, c^{(*)} R\right) \rightarrow$ $(D, P, Q)$ for any $g: R \rightarrow P * Q$.

\section{Abstract relational model}

The purpose of this section is to propose an abstract relational model which is a meta-model of various database models such as relational, network and hierarchical model.

Theorem 1 holds when we change each $\boldsymbol{S e t}^{A}$ to a functor category $\boldsymbol{D}^{A}$ where $\boldsymbol{D}$ is a category with direct products.

Furthermore, we can generalize the relational model of Section 2 to generate an abstract relational model.

\subsection{Definition of abstract relational model}

Definition 3.1. An abstract relational model is a quadruple $\boldsymbol{M}=(\boldsymbol{S}, \boldsymbol{T}, \boldsymbol{O}, T)$ where

(i) $\boldsymbol{S}$ is a category called the category of schemata.

(ii) for each $A \subseteq O b S$, there are a category $\boldsymbol{T}^{A}$ of types (or of domains), a caregory 
$O^{A}$ of occurrences on $A$ and a functor $T^{A}: O^{A} \rightarrow T^{A}$,

(iii) for each $a: A \rightarrow B \in$ Mor $\boldsymbol{S}$, there are the projection functor of types $a^{*}: \boldsymbol{T}^{B} \rightarrow \boldsymbol{T}^{\boldsymbol{A}}$ and the projection functor of occurrences $a^{(*)}: \boldsymbol{O}^{B} \rightarrow \boldsymbol{O}^{A}$, subject to the following conditions

(1) $i d_{A}^{*} \cong I d_{T^{A}}, i d_{A}^{(*)} \cong I d_{o^{A}}(A \in O b S)$,

(2) $(b \circ a)^{*} \cong a^{*} \circ b^{*},(b \circ a)^{(*)} \cong a^{(*)} \circ b^{(*)}$ (if $b \circ a$ is defined in $\left.\boldsymbol{S}\right)$,

(3) $a^{*} \circ T^{B} \cong T^{A} \circ a^{(*)}(a: A \rightarrow B \in \operatorname{Mor} \boldsymbol{S})$,

(4) $a^{*}$ has the right adjoint $a_{*}(a \in \operatorname{Mor} S)$.

We can say $\boldsymbol{T}$ and $\boldsymbol{O}$ are $\boldsymbol{S}$-indexed categories and $T: \boldsymbol{O} \rightarrow \boldsymbol{T}$ is a $\boldsymbol{S}$-indexed functor except that $\boldsymbol{S}$ may not have finite limits [5].

\subsection{Decomposition and natural join in abstract relational models}

Let $M=(\boldsymbol{S}, \boldsymbol{T}, \boldsymbol{O}, T)$ be an abstract relational model. We suppose the diagram Fig. 3.1. of $\boldsymbol{S}$ similarly to Section 2.2 .

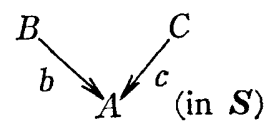

Fig. 3.1.

DEFINITION 3.2. $\left((b, c)\right.$-compatible occurrence) The category $\boldsymbol{O}^{b: c}$ of $(b, c)$-compatible occurrences is defined as follows.

(i) an object is a triple $(D, P, Q)$ where $D \in O b \boldsymbol{T}^{A}, P \in O b \boldsymbol{O}^{B}$ and $Q \in O b O^{C}$ such that $T^{B} P \cong b^{*} D$ and $T^{C} Q \cong c^{*} D$,

(ii) a morphism $f:(D, P, Q) \rightarrow\left(D^{\prime}, P^{\prime}, Q^{\prime}\right)$ is a triple $f=(g, h, k)$ where $g: D \rightarrow D^{\prime}$ $\in \operatorname{Mor} \boldsymbol{T}^{A}, h: P \rightarrow P^{\prime} \in \operatorname{Mor} \boldsymbol{O}^{B}$ and $k: Q \rightarrow Q^{\prime} \in \operatorname{Mor} \boldsymbol{O}^{C}$ such that the following diagrams are commutative
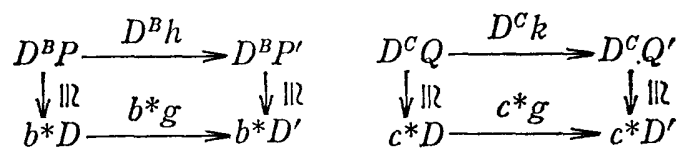

Decomposition functors are defined similarly to Definition 2.6.

Definition 3.3. $\left((b, c)\right.$-decomposition functor) The $(b, c)$-decomposition functor Dec $c^{b ; c}$ is a functor defined by the following diagram,

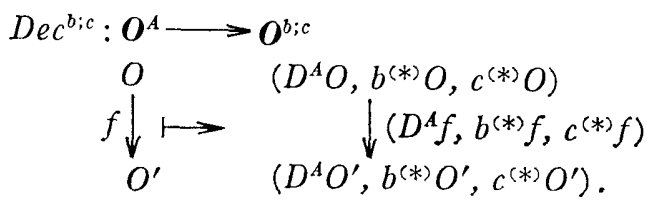

Natural join functors is defined using Theorem 1. 
Definition 3.4. ((b, $c)$-natural join functor) If the $(b, c)$-decomposition functor $D e c^{b ; c}: \boldsymbol{O}^{A} \rightarrow \boldsymbol{O}^{b ; c}$ has the right adjoint, then we call this right adjoint functor $(b, c)$ national join functor and write as

$$
J \operatorname{inn}^{b ; c}: \boldsymbol{O}^{b ; c} \rightarrow \boldsymbol{O}^{A} .
$$

$J_{o i n}^{b ; c}(D, P, Q)\left((D, P, Q) \in O b O^{b ; c}\right)$ and $\operatorname{Join}^{b ; c}(g, h, k)\left((g, h, k) \in\right.$ Mor $\left.\boldsymbol{O}^{b ; c}\right)$ are written as $P * Q$ and $h * k$ respectively for short.

We prove a lemma before Theorem 2 which shows a sufficient condition for an abstract relational model to have natural joins.

LEMMA 3.1. Let $b^{*}, c^{*}, b^{(*)}, c^{(*)}, T^{A}, T^{B}$ and $T^{C}$ have the right adjoints $b_{*}, c_{*}, b_{(*)}$, $c_{(*)}$, Fill $^{A}$, Fill $^{B}$ and Fill ${ }^{C}$ respectively, i.e.,

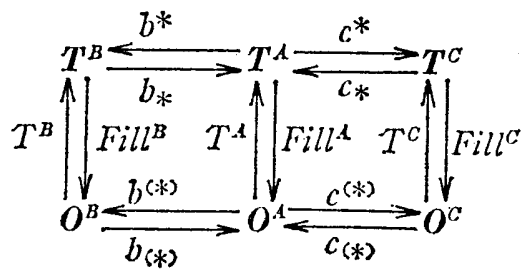

Then, for any $(D, P, Q) \in O b O^{b ; c}$, we have

(1) $b_{(*)} F_{i l l}^{B} T^{B} P \cong F_{i l l}^{A} b_{*} b^{*} D$

(2) $c_{(*)}$ Fill $^{C} T^{C} Q \cong$ Fill $^{C} c_{*} c^{*} D$.

Proof. We prove only (1). (2) is proved similarly.

Let natural transformations $\eta^{B}: I d_{0^{B} \rightarrow F i l l^{B} T^{B}}$ and $e^{b}: I d_{T^{A} \rightarrow b_{*} b^{*}}$ be units of adjoint pairs $T^{B} \dashv F i l l^{B}$ and $b^{*} \dashv b_{*}$ respectively. From the properties of adjoint functors, $b^{*} T^{A}$ is the left adjoint of Fill $^{A} b_{*}$ and $T^{B} b^{(*)}$ is the left adjoint of $b_{(*)}$ Fill $^{B}$. However, $b^{*} T^{A} \cong T^{B} b^{(*)}$ is assumed in Definition 3.1. Therefor, we have Fill $b_{*} \cong b_{(*)} F_{i l l}{ }^{B}$ from the uniqueness of a rigt adjoint. (1) is satisfied since $T^{B} P \cong b^{*} D$.

THEOREM 2. An abstract relational model $M$ has natural joins if

(1) for each $S \in O b S, T^{S}: \boldsymbol{O}^{S} \rightarrow \boldsymbol{T}^{\boldsymbol{S}}$ has the right adjoint Fill $\boldsymbol{T}^{\boldsymbol{S}} \rightarrow \boldsymbol{O}^{S}$ and $\boldsymbol{O}^{S}$ has pullbacks, and

(2) for each $s: S \rightarrow S^{\prime} \in M o r ~ S, s^{(*)}: \boldsymbol{O}^{S^{\prime}} \rightarrow \boldsymbol{O}^{S}$ has the right adjoint $s_{(*)}: \boldsymbol{O}^{S} \rightarrow \boldsymbol{O}^{S^{\prime}}$.

To prove this theorem, we use a property about adjoint functors that if $F, F^{\prime}$ : $\boldsymbol{D} \rightarrow \boldsymbol{C}$ are functors with the right adjoint functors $G, G^{\prime}: \boldsymbol{C} \rightarrow \boldsymbol{D}$ respectively and $\rho: F^{\prime} \rightarrow F$ is a natural transformation, then there is a natural transformation $\tau: G \rightarrow G^{\prime}$ such that

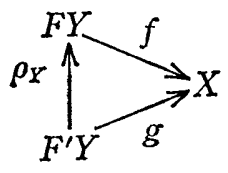

commutes if and only if 


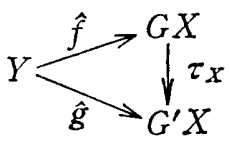

commutes where $f$ and $g$ are corresponding morphisms of $f$ and $g$ by adjoint pairs $F \dashv G$ and $F^{\prime} \dashv G^{\prime}$. Specially, if $\rho$ is isomorphic, then $\tau$ is also isomorphic (e.g. [10, See 16.4.3]).

Proof of Theorem 2. Let $(D, P, Q)$ be an object of $\boldsymbol{O}^{b ; c}$. We define $P * Q$ by Fig. 3.2 using isomorphisms in Lemma 3.1.

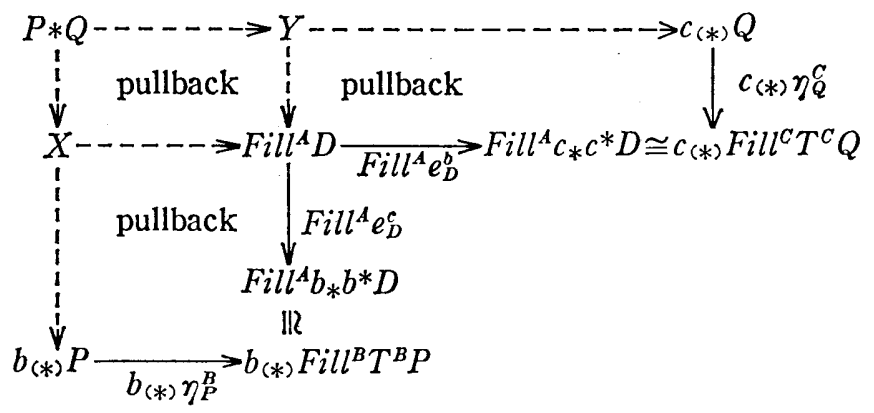

Fig. 3.2.

in Fig. 3.2, $\eta^{B}, \eta^{C}, e^{b}$ and $e^{c}$ are units of adjoint pairs $T^{B} \dashv F_{i l l}^{B}, T^{C} \dashv F i l l^{C}, b^{*} \dashv b_{*}$ and $c^{*} \rightarrow c_{*}$ respectively.

Let $W$ be the subdiagram of the diagram in Fig. 3.2. which is represented by solid arrows.

Pullbacks in Fig. 3.2 make $P * Q$ the limit of $W$. Notice that there is a bijective correspondence between a morphism $f: R \rightarrow P * Q$ and a triple $\left(l: R \rightarrow\right.$ Fill $^{A} D, m: R \rightarrow b_{(*)} P$, $\left.n: R \rightarrow c_{(*)} R\right)$ such that the diagram of Fig. 3.3 commutes since $P * Q=\lim W$. We define $Z$ as

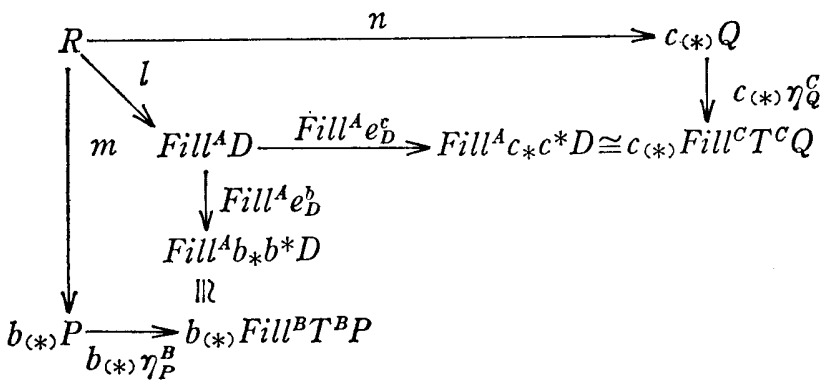

Fig. 3.3.

the set of all the triples $(l, m, n)$ such that Fig. 3.3 commutes. 
It is sufficient for proving this theorem to show $Z \cong \boldsymbol{R}^{b ; c}\left(D e c^{b ; c} R,(D, P, Q)\right)$.

Let $(g, h, k):\left(T^{A} R, b^{(*)} R, c^{(*)} R\right) \rightarrow(D, P, Q)$ be in $R^{b ; c}$. We define $l: R \rightarrow$ Fill $^{A} D$, $m: R \rightarrow b_{(*)} P$ and $n: R \rightarrow c_{(*)} Q$ by
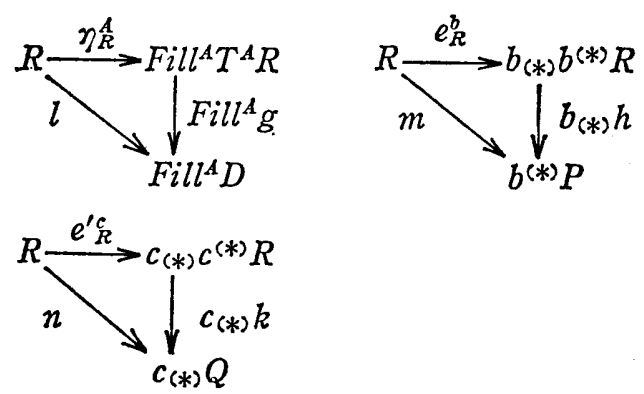

where $e^{\prime b}: I d_{0^{A} \rightarrow b_{(*)}} b^{(*)}$ and $e^{c}: I d_{0^{A} \rightarrow c_{(*)} c^{(*)}}$ are units of adjoint pairs $b^{(*)} \rightarrow b_{(*)}$ and $c^{(*)} \rightarrow c_{(*)}$ respectively.

First, we prove that the following diagram commutes:

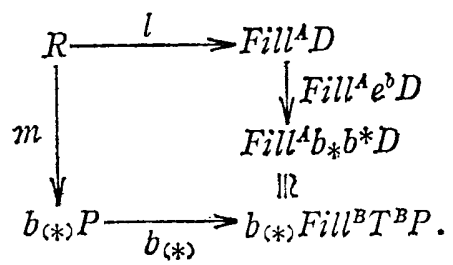

Let $s: T^{B} b^{(*)} R \rightarrow b^{*} D$ be a morphism in $\boldsymbol{T}^{B}$ such that

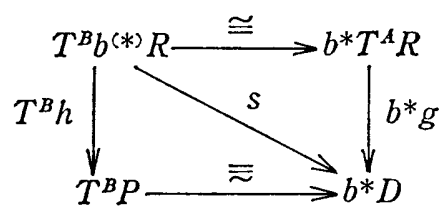

commutes.

From properties of adjoint functors, the diagrams of Fig. 3.4 are all commutative. In Fig. 3.4, operators " $\wedge$ " and " $\sim$ " are the adjunction isomorphisms of $F i l l^{A} \rightarrow T^{A}$ (or Fill $\left.^{B} \rightarrow T^{B}\right)$ and $b^{*} \dashv b_{*}$ respectively. 

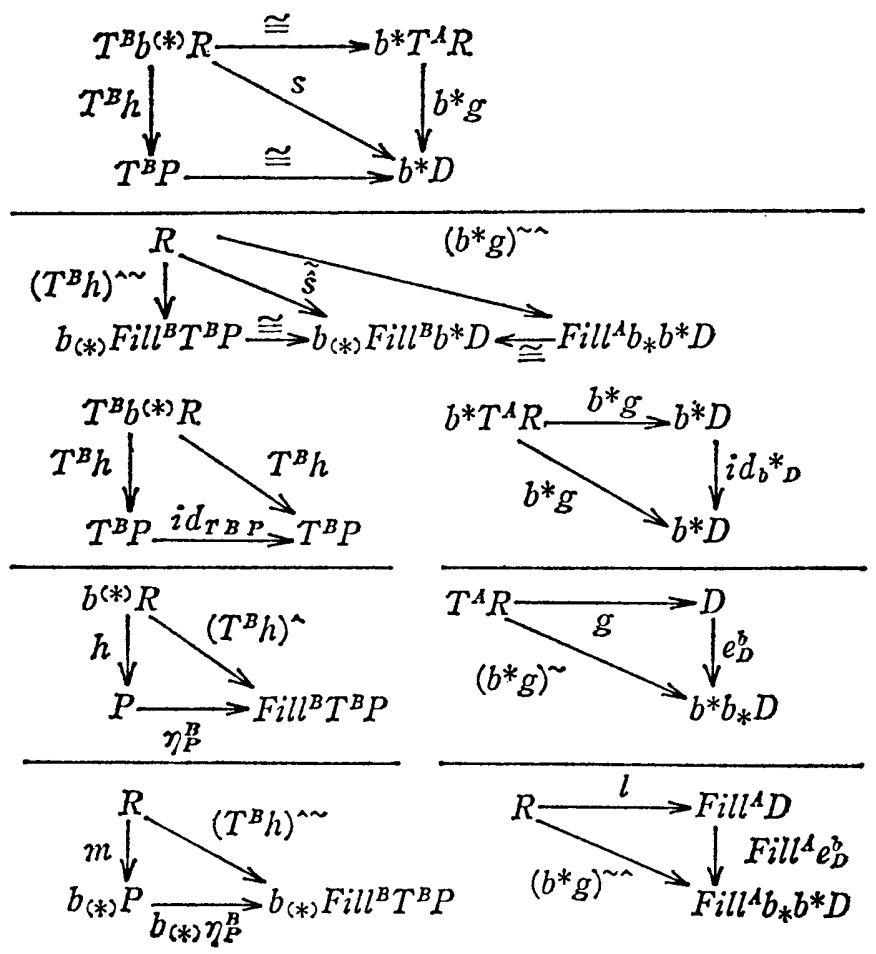

Fig. 3.4.

Therefore, we have

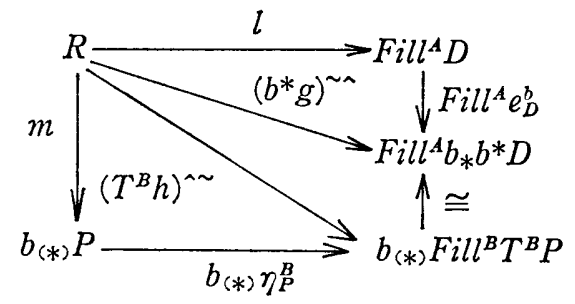

Similarly, the following diagram commutes:

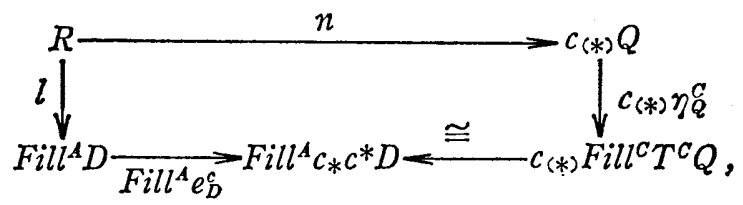

that is, $(l, m, n) \in Z$.

Obviously, this correspondence $(g, h, k) \rightarrow(l, m, n)$ is naturally bijective. (q.e.d.) 


\subsection{Examples of abstract reational models}

(1) Relational Data Model

We have the relational model when $\boldsymbol{S}$ is $\boldsymbol{S e t}$ and for each (attribute) set $A$, Set, $\boldsymbol{T}^{A}, \boldsymbol{O}^{A}$ and $T^{A}$ are $\boldsymbol{S e t}{ }^{A}, \boldsymbol{R}^{A}$ and $D^{A}$ of Section 2 respectively. For a function $a: A \rightarrow B$ between attribute sets and $D=\left\{D_{i}\right\}_{i \in A} \in O b \boldsymbol{S e t}^{A}, a_{*} D$ is the family $\left\{\prod_{a(i)=j} D_{i}\right\}_{j \in B}$.

(2) Network Model

We define a simple network model $\boldsymbol{M}=(\boldsymbol{S}, \boldsymbol{T}, \boldsymbol{O}, T)$ as an abstract relational model.

(i) $\boldsymbol{S}$ is the category of multigraphs, i. e., Bachman's diagrams $G=(S, R, \Delta: S \rightarrow$ $R \times R$ ) where $S$ is a set of set-types, $R$ is a set of record-types and $\Delta$ assigns a set-type $s$ to a pair (own $(s)$, mem $(s)$ ) of owner and member record-types. A morphism $f:(S, R, \Delta) \rightarrow\left(S^{\prime}, R^{\prime}, \Delta^{\prime}\right)$ is a pair $f=\left(f^{S}: S \rightarrow S^{\prime}, f^{R}: R \rightarrow R^{\prime}\right)$ such that

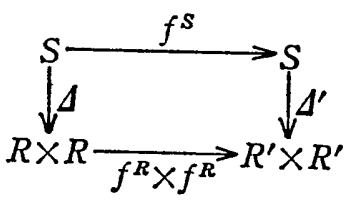

commutes.

(ii) For $G=(S, R, \Delta) \in O b S, \boldsymbol{T}^{G}$ is the category $\boldsymbol{S e t}^{R}$ whose object $\left\{V_{r}\right\}_{r \in R}$ is the family of value sets of record-types.

(iii) The occurrence category $\boldsymbol{O}^{G}(G=(S, R, \Delta) \in O b S)$ is defined as follows:

(a) an object is $N=\left(\left\{V_{r}\right\}_{r \in R},\left\{X_{r}\right\}_{r \in R},\left\{v_{r}: X_{r} \rightarrow V_{r}\right\}_{r \in R},\left\{o_{s}: X_{m e m(s)} \rightarrow X_{o w n(s)}\right\}_{s \in S}\right)$ where $\left\{V_{r}\right\}_{r \in R} \in O b \boldsymbol{T}^{G},\left\{X_{r}\right\}_{r \in R}$ is a family of record sets, $v_{r}: X_{r} \rightarrow V_{r}$ assigns a record $x$ to its value and $o_{s}: X_{m e m(s)} \rightarrow X_{o w n(s)}$ assigns a member record to its owner record,

(b) a morphism $g: N \rightarrow N^{\prime}\left(N=\left(\left\{V_{r}\right\}_{r \in R},\left\{X_{r}\right\}_{r \in R},\left\{v_{r}\right\}_{r \in R},\left\{o_{s}\right\}_{s \in S}\right), N^{\prime}=\left(\left\{V_{r}^{\prime}\right\}_{r \in R}\right.\right.$, $\left.\left.\left\{X_{r}^{\prime}\right\}_{r \in R},\left\{v_{r}^{\prime}\right\}_{r \in R},\left\{o_{s}^{\prime}\right\}_{s \in S}\right)\right)$ is a pair $g=\left(\left\{g_{r}^{V}: V_{r} \rightarrow V_{r}^{\prime}\right\}_{r \in R},\left\{g_{r}^{X}: X_{r} \rightarrow X_{r}^{\prime}\right\}_{r \in R}\right)$ such that $g_{r}^{V} \circ v_{r}=v_{r}^{\prime} \circ g_{r}^{X}(r \in R)$ and $g_{m e m(s)}^{X} \circ o_{s}=o_{s}^{\prime} \circ g_{o w n}^{X}(s)$.

(iv) Let $f: G \rightarrow G^{\prime}\left(G=(S, R, \Delta), G^{\prime}=\left(S^{\prime}, R^{\prime}, \Delta^{\prime}\right)\right)$ be in $\boldsymbol{S} . f^{*}: \boldsymbol{T}^{G^{\prime}} \rightarrow \boldsymbol{T}^{G}$ is equal to $\left(f^{R}\right)^{*}: \boldsymbol{S e t} \boldsymbol{t}^{R^{\prime}} \rightarrow \boldsymbol{S e t} \boldsymbol{t}^{R}$ defined as in Section 2. $f_{*}: \boldsymbol{T}^{G \rightarrow \boldsymbol{T}^{G^{\prime}}}$ is equal to $\left(f^{R}\right)_{*}: \boldsymbol{S e} \boldsymbol{t}^{R \rightarrow}$ $\boldsymbol{S e t}^{R^{\prime}}$ defined as in the above example (1). $f^{(*)}: \boldsymbol{O}^{G^{\prime}} \rightarrow \boldsymbol{O}^{G}$ assigns $N=\left(\left\{V_{r^{\prime}}\right\}_{r^{\prime} \in R^{\prime}}\right.$, $\left.\left\{X_{r^{\prime}}\right\}_{r^{\prime} \in R^{\prime}},\left\{v_{r^{\prime}}\right\}_{r^{\prime} \in R^{\prime}},\left\{o_{s^{\prime}}\right\}_{s^{\prime} \in S^{\prime}}\right) \in O b O^{G^{\prime}}$ to $\left(\left\{V_{f^{R}(r)}\right\}_{r \in R},\left\{X_{f} R_{(r)}\right\}_{r \in R},\left\{v_{f} R_{(r)}\right\}_{r \in R},\left\{o_{f} S_{(s)}\right\}_{s \in S}\right)$ and assigns $g=\left(\left\{g_{r^{\prime}}^{V}\right\}_{r^{\prime} \in r^{\prime}},\left\{g_{r^{\prime}}^{X}\right\}_{r^{\prime} \in R^{\prime}}\right) \in \operatorname{Mor} \boldsymbol{T}^{G^{\prime}}$ to $\left(\left\{g_{f}^{V_{(r)}}\right\}_{r \in R},\left\{g_{f}^{X} R_{(r)}\right\}_{r \in R}\right)$.

(v) $T^{G}: \boldsymbol{O}^{G} \rightarrow \boldsymbol{T}^{G}(G=(S, R, \Delta))$ assigns $\left(\left\{V_{r}\right\}_{r \in R}, X, v, o\right) \in O b \boldsymbol{O}^{G}$ to $\left\{V_{r}\right\}_{r \in R}$ and assigns $\left(\left\{g_{r}^{V}\right\}_{r \in R},\left\{g_{r}^{X}\right\}_{r \in R}\right)$ to $\left\{g_{r}^{V}\right\}_{r \in R}$.

(3) Hierarchical Model

The hierarchical model can be obtained restricting $\boldsymbol{S}$ of the network model in (2) to the category of trees.

The above examples all satisfy the condition of Theorem 2, that is, they have natural joins. We omit its proof. In the examples (2) and (3), notice that each category $\boldsymbol{O}^{G}$ of occurrences is equivalent to a certain $\boldsymbol{S e t}$-valued functor category $\boldsymbol{S e t}^{\widetilde{G}}$. 


\section{Conclusion}

We have proposed an abstract relational model which is a meta-model for database models such as relational, network and hierarchical models.

A natural join functor has been defined as the right adjoint of a decompositon functor. This definition has been obtained by the result that a natural join is the right adjoint of a decomposition in a relational database model on the category of sets.

As an application, a sufficient condition has been proved for a database model to have natural joins.

We can expect that concepts of the relational database models such as dependencies, normal forms, algebras are disscussed independently of database models in our approach.

Another topic that requires further research is semantics of an astract relational model.

\section{Acknowledgements}

In preparing this paper, the author is grateful to Dr. Tosio Kjtagawa, Director of the International Institute for Advanced Study of Social Information Science for his encouragement and advices, to Dr. Kaname Kobayasi, Mr. Hajime Sawamura and Mr. Taku Takesima of the insttitute for their valuable discussions and to Assistant Professor Dr. Yasuo Kawahara of Kyushu University, Mr. Yuzuru Tanaka of Hokkaido University and Mr. Tatsunari Mizukami of Kusiro Technical College for useful discussions.

\section{References}

[1] Chex, P.P.S.: The Etity-Relationship Model-Toward a Unified View of Data, ACM Trans. on Database Systems, Vol. 1, No. 1 (1976), 9-36.

[2] Codd, E.F.: Relational Model of Data for Larde Shared Data Banks, CACM, Vol. 13, No. 6 (1970), 377-387.

[3] Codd, E.F.: Relational Completeness of Database Sublanguages, Courant Computer science Symposia 6: Database Systems, Prentice Hall (1972), 65-98.

[4] DAte, C.J.: An Introduction to Database Systems, Addison-Wesley (1975).

[5] Johnstone, P.T. and Paré, R., ed.: Indexed Categories and Their Applications, Lec. Notes in Math. 661, Springer-Verlag (1978).

[6] Kato A.: Projection/Join Functors and Decomposability of Relations, Int. Inst. for Advanced Study of Soc. Inf. Sci., Fujitsu Ltd., Research Report No. 22 (1981).

[7] Kawahara, Y.: Ken ni okeru Chusho Database ni tsuite (On Abstract Databases in Categories), unpublished, in Japanese (1981).

[8] Manes, E.G., ed.: Category Theory Applied to Computation and Control, Lec. Notes in Comp. Sci. 25, Springer-Verlag (1975).

[9] Mizukami, T.: Data Base Semantics Based on Intuitionistic Logics, to appear.

[10] Shubelt, H.: Categories, (translated from German by E. Gray), Springer-Verlag (1972).

[11] TanakA, Y.: Information Space Model, Proc. Formal Bases for Data Bases (Toulous, 1979).

Communicated by T. Kitagawa

Received November 8, 1982 